\title{
AN EXTENDED LINEARIZED NEURAL STATE SPACE BASED MODELING AND CONTROL"
}

\author{
Qing WU ${ }^{1}$, Yongji WANG ${ }^{1}$, and Hong WANG ${ }^{2}$ \\ ${ }^{1}$ Dept. of Control Science and Engineering, CNCS, \\ Huazhong University of Science and Technology, 430074, Wuhan, P. R. China \\ Email: yjwang@public.wuhan.cngb.com \\ ${ }^{2}$ Dept. of Paper Science, UMIST, Manchester, M60 1QD, UK \\ Email: Hong.wang@umist.ac.uk
}

\begin{abstract}
In this paper, an extended linearized neural state space (ELNSS) topology is proposed, where an ELNSS based modeling and control strategy for a class of nonlinear systems is presented. In terms of the modeling, the extended Kalman filter (EKF) algorithm is used to train the parameters inside the ELNSS model, where a high order correlation method is applied to validate the estimated model. This is then followed by the design of a one-step-ahead predictive controller for affine nonlinear systems. The stability of the so-formed closed loop control system is investigated, and several sufficient conditions that guarantee the local asymptotic stability are established. Two simulation examples are used to demonstrate the proposed algorithm and desired results have been obtained. Copyright $@ 2002$ IFAC
\end{abstract}

Keywords: neural networks, state space, predictive control, nonlinear systems, asymptotic stability

\section{INTRODUCTION}

Since neural networks are powerful approximation tools, in recent years they have been used to the modeling and control of unknown, or partially unknown, nonlinear dynamic systems. A number of architectures for identification and control have been developed and examples are multilayer perceptron (MLP) and radial basis function (RBF) networks. In the neural network based control system design, the primary problem is to construct a neural network of a suitable type and architecture. Once this has been solved, the controller design and system analysis can be readily carried out.

*This works is supported by NSFC under grant 69974017 , NSFC two-base international collaborative program (6010720098), SFC Guangxi China (0135065), the CSC returned scholar foundation, key teacher project of Ministry Education of China and Key laboratory of Ministry Education of China for Image processing and Intelligent control.
In 1995 Suykens et al presented a neural state space model for the identification of nonlinear system. From then on, many researchers adopted this neural state space structure to model and design controllers of nonlinear systems (Liang et al.,1999; Pavel et al. 1999; Zamarren et al. 2000; Zhao et al., 1998; Zhao et al., 1999a; Zhao et al., 1999b). Based upon these formulations, recently a pseudo-linear neural network (PNN) was presented (Wang et al. 1999a; 1999b), where the scheme for the system modeling has been developed. Both the one-step and the multi-step predictive controllers of a typical chemical process were made. It has been shown that the convergence of PNN training process can be guaranteed, and the asymptotic stability of the closed loop PNN-based control system has been established, where some sufficient conditions, that guarantee the closed loop stability, were obtained.

Based on the PNN model, an extended linearized neural state space model (ELNSS) is proposed in this paper. The ELNSS is composed of multi-layers neurons, where the first hidden and output layers are 
the same as those used in a standard MLP neural network. In specific, the second hidden layer consists of neurons with algebraic multiplications, whose outputs are the products of the inputs of ELNSS and the outputs of the first hidden layer.

The problem to be solved here is whether this new type of neural state space model can approximate arbitrary nonlinear discrete dynamic systems in the same way as that of an MLP neural network. In this paper, the existence of ELNSS as a universal approximator to a class of nonlinear systems is proved under some conditions. The parameter identification for the ELNSS employs the wellknown extended Kalman filter (EKF) algorithm. This is the same as the situation when the EKF is applied to the training of the MLP neural network. It has been shown that the convergence of the training algorithm can be guaranteed if the associated learning rate is correctly chosen.

It is well-known that the one-step-ahead predictive control is an effective scheme in linear adaptive control. For nonlinear systems, a one-step-ahead controller using a MLP neural network was proposed by Tan and Cauwenberghe (1996), where the gradient-based optimization was adopted. Since the neural state space model exhibits a quasilinear character, the one-step-ahead controller design can also be applied to the ELNNS models. In this paper, the stability analysis of the ELNSS-based one-stepahead predictive control system is proposed using the well-known Lyapunov theory, and the sufficient conditions for the local asymptotic stability of the ELNSS control system are derived.

The proposed approaches are illustrated with two simulated examples, including the control of an affine nonlinear system. Desired closed loop responses are obtained.

The paper is organized as follows. The proposed ELNSS model, its parameters identification and the convergence analysis are described in Section 2. The design of an ELNSS-based one-step-ahead predictive controller presented in Section 3, where the closed loop stability is also analyzed. This is followed by the presentation of some simulation results in Section 4. Finally, conclusion is given in Section 5.

\section{THE EXTENDED LINEARIZED NEURAL STATE SPACE MODEL AND ITS MODELING}

\subsection{The architecture of the ELNSS}

Consider the nonlinear dynamic system

$$
\begin{aligned}
& \dot{x}(t)=f(x(t), u(t)) \\
& y(t)=h(x(t))
\end{aligned}
$$

where $x$ is the state vector, $u$ is the input and $y$ is the output. This is a general nonlinear expression and its direct control formulation is difficult to perform. To overcome this difficulty, Friendland (1996) proposed the following extended linearization model of nonlinear system

$$
\begin{aligned}
& \dot{x}=A(x) x+B(x) u \\
& y=C x
\end{aligned}
$$

where $A(x)$ and $B(x)$ are general nonlinear functions of the state $x$. In this paper, only model (2) will be used.

For system (2), Wang et al (1999a) presented a pseudo-linear neural network (PNN), where the system modeling and a one-step and multi-step predictive control of a continuous stirred tank reactor (CSTR) were successfully performed. Based on the PNN, an extended linearized neural space state model can be established, which is a recurrent network model of the following form

$$
\begin{aligned}
& X(t+1)=A(X(t)) X(t)+B(X(t)) U(t) \\
& Y(t)=C X(t) \\
& A(X(t))=\sigma\left(X(t), W_{a}\right) \\
& B(X(t))=\sigma\left(X(t), W_{b}\right) \\
& \sigma(x)=\frac{1-\exp (x)}{1+\exp (x)}
\end{aligned}
$$

where, $X(k), Y(k)$ and $U(k)$ are state, output and input, respectively, and $\sigma(\bullet)$ is a sigmoid function.

Consider an SISO nonlinear system and suppose that matrix $A$ is of a controllable companion canonical form, then the architecture of the ELNSS is shown in Figure 1.

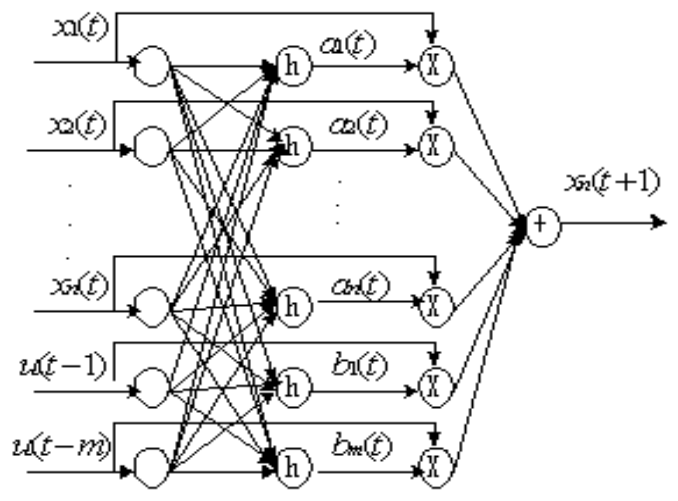

Fig.1 The architecture of ELNSS

Different from the widely used multiplayer perceptrons (MLP), the "X circle" in Fig.1 symbolizes the multiplication neurons, which perform the algebraic multiplications between the input of ELNSS and the output of the first hidden layer. In addition, "+" symbolizes the algebraic sum and " $h$ " stands for the well-known sigmoid functions. At each sample time $t$, the inputs of ELNSS are $I(t)=\left[x_{l}(t), . x_{n}(t), u(t-1), \ldots u(t-m)\right]^{T}$, and $\left\{a_{j}(t), b_{j}(t)\right\}$ are the outputs of the $j$-th of the first hidden neurons. $x_{n}(t+1)$ is the output of the network and $y_{m}(t)$ is the output of system. $W$ in Fig. 1 represents the weights from the input layer to the first hidden layer. The 
input-output relationship of such an ELNSS can be formulated to give:

$$
\begin{aligned}
& X(t+1)=A X(t)+B U(t) \\
& Y_{m}(t)=C X(t)
\end{aligned}
$$

where $X(t)=\left[x_{1}(t), x_{2}(t), \ldots, x_{n}(t)\right]^{T}$ is $n$th order state vector, $U(t)=[u(t-1), \ldots, u(t-m)]^{T}$ is $m$ th order control vector and parameter matrices are of the following form:

$$
\begin{aligned}
A(\bullet) & =\left[\begin{array}{ccccc}
0 & 1 & 0 & \cdots & 0 \\
\vdots & 0 & 1 & \cdots & 0 \\
\vdots & \vdots & \vdots & \cdots & 1 \\
a_{1}(t) & \cdots & \cdots & \cdots & a_{n}(t)
\end{array}\right] B(\bullet)=\left[\begin{array}{c}
b_{1}(t) \\
\vdots \\
\vdots \\
b_{n}(t)
\end{array}\right](5) \\
C & =\left[\begin{array}{llll}
c_{1} & \cdots & \cdots & c_{n}
\end{array}\right]
\end{aligned}
$$

The advantage of ELNNS models is that each element of $A$ or $B$ may be the combination of linear part $\left\{a_{i}, b_{i}\right\}$ and nonlinear time varying part $\left\{a_{i}{ }^{\prime}(t), b_{i}{ }^{\prime}(t)\right\}$. As a result, the well-established linear system approaches can be readily extended to the controller design for the ELNSS represented system.

\subsection{Parameters identification of the ELNSS}

The extended Kalman filtering (EKF) algorithm (Gintaras and Lee, 1994) is adopted in training the ELNSS model. Since the learning rate in EKF can be adapted, a proper tuning of the learning rate can be naturally realized for the gradient-based search scheme. This ensures a fast convergence for the training algorithm.

The principle of EKF is as follows:

Considering the nonlinear system described by (1) that is modeled by an ELNNS. Denote the parameters of the ELNNS as $\theta$, and then the nonlinear state space equation of the ELNNS can be rewritten as

$$
\begin{aligned}
& \theta(t+1)=\theta(t) \\
& y_{m}(t)=f(\theta(t))+v(t)
\end{aligned}
$$

where $v(t)$ is a noise.

For system (6)-(7), the EKF learning algorithm for updating the parameters of the ELNNS is given by

$$
\begin{aligned}
& \theta(t)=\theta(t-1)+\eta(t) K(t)\left(y(t)-y_{m}(t)\right) \\
& K(t)=P(t) H(t)\left[\left(H^{T}(t) P(t) H(t)+R(t)\right)\right]^{-1} \\
& P(t+1)=P(t)-K(t) H^{T}(t) P(t)
\end{aligned}
$$

where $K(t), P(t)$ and $R(t)$ are matrices as used in the Kalman filter and $\eta(t)$ is the learning rate. In the SISO system, $R(t)=r(t)$, which is in fact an estimate of covariance of noise $v(t)$. In this case, we have

$$
\begin{aligned}
& r(t)=r(t-1)+\mu(t)\left[\left(y(t)-y_{m}(t)\right)^{2}-r(t-1)\right] \\
& \mu(t)=1 / t
\end{aligned}
$$

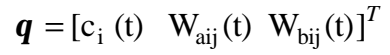

$$
\begin{aligned}
& P_{i j}(t)=\frac{\partial y(t)}{\partial W_{a i j}} \quad Q_{i j}(t)=\frac{\partial y(t)}{\partial W_{b i j}}
\end{aligned}
$$

$$
\begin{aligned}
& P_{i j}(t)=\sigma^{\prime}\left(a_{i}(t-1)\right) \hat{x}_{i}(t-1) \hat{x}_{j}(t-1) \\
& +a_{n}(t+1) P_{i j}(t-1) \\
& Q_{i j}(t)=c_{i} \sigma^{\prime}\left(b_{i}(t-1)\right) \hat{x}_{j}(t-1) u(t-1) \\
& +c_{n} a_{n}(t-1) \sigma^{\prime}\left(b_{i}(t-2)\right) \hat{x}_{j}(t-2) u(t-2) \\
& H(t)=\left[\frac{\partial y(t)}{\partial \theta}\right]_{\theta=\theta(t-1)} \\
& =\left\{\begin{array}{cc}
\hat{x}_{j}(t-1) & \text { if } \theta_{j}=c_{j} \\
c_{n} P_{i j}(t) & \text { if } \theta_{j}=W_{a i j} \\
Q_{i j}(t) & \text { if } \theta_{j}=W_{b i j}
\end{array}\right.
\end{aligned}
$$

\subsection{The Universal Approximation Ability of ELNSS}

It is well known that the MLP neural network, with at least one hidden layer, can approximate arbitrary continuous nonlinear functions defined on a compact set. As a result, it can be used as a universal nonlinear discrete dynamic model (Liu and Feng, 1995). When the PNN is used to model a nonlinear discrete dynamic system, its universal approximation ability was also proved (Wang et al., 1998a, 1999a). Because ELNSS is an extension of PNN, its approximation ability to a nonlinear discrete-time model can also be justified. This means that ELNSS can approximate, at a pre-specified accuracy, any nonlinear dynamic systems (Wang et al, 1998b).

\subsection{Convergence of the training process of ELNSS}

With respect to the convergence of learning process, the following theorem can be established.

Theorem 1: The convergence of ELNSS can be guaranteed if $\eta(t)$ in equation (8) is chosen as

$$
0<\eta(t)<\frac{2}{g_{\max }}
$$

where

$$
\begin{gathered}
g_{\text {max }}=\max \left|g_{m}(t)\right|=\max _{t}\left|H^{T}(t) P(t) H(t) A(t)\right| \\
A(t)=\left[R(t)+H^{T}(t) P(t) H(t)\right]^{-1}
\end{gathered}
$$

The optimal $\eta$ is

$$
\eta^{*}=1 / g_{\max }
$$

Proof:

By choosing the Lyapunov function as

$$
V(t)=\frac{1}{2} e^{T}(t) e(t)
$$

and approximating the modeling error as

$$
\begin{aligned}
& \Delta e(t)=\left[\frac{\partial e(t)}{\partial \theta}\right]^{T} \Delta \theta \\
& =-H^{T}(t) P(t) H(t) A(t) e(t) \\
& =-\eta(t) g_{m}(t) e(t)
\end{aligned}
$$

it can be shown that

$$
\begin{aligned}
& \Delta V(t)=\Delta e(t)\left[e(t)+\frac{1}{2} \Delta e(t)\right] \\
& =-\eta(t) g_{m}(t)\left[1-1 / 2 \eta(t) g_{m}(t)\right] e^{2}(t)
\end{aligned}
$$


If (18) is satisfied, then $\Delta V(t)<0$, which guarantees that the predictive error $e(t)$ is decreasing and the training process is convergent.

\subsection{Validation of ELNSS Models}

A number of model validity tests for nonlinear model identification procedures have been developed. Typical examples are the statistical chi-square test, the Akaike Information Criterion (AIC) (Ljung, 1987), the predicted squared error criterion (Zhao et al. 1999a) and the high-order correlation tests (Billings and Voon, 1986). The validity of an ELNSS model can be assessed using the following highorder correlation tests:

$$
\begin{array}{ll}
\Phi_{\varepsilon \varepsilon}=E[\varepsilon(t-\tau) \varepsilon(t)]=\delta(\tau) & \forall \tau \\
\Phi_{u \varepsilon}=E[u(t-\tau) \varepsilon(t)]=0 & \forall \tau \\
\Phi_{u^{2} \varepsilon}=E\left\{\left[u^{2}(t-\tau)-\overline{u^{2}(t)}\right] \varepsilon(t)\right\}=0 & \forall \tau \\
\Phi_{u^{2} \varepsilon^{2}}=E\left\{\left[u^{2}(t-\tau)-\overline{u^{2}(t)}\right] \varepsilon^{2}(t)\right\}=0 & \forall \tau \\
\Phi_{e(e u)}=E[\varepsilon(t) \varepsilon(t-1-\tau) u(t-1-\tau)]=0 & \forall \tau
\end{array}
$$

where $\varepsilon$ is the model residual and $\overline{u^{2}}$ is the time average of $u^{2}$. These tests look into the cross correlation amongst model residuals and inputs.

Normalization should be carried out to give all test data a range between \pm 1 . Also, the approximation should be performed at $95 \%$ confidence bounds for $1.96 / \sqrt{N}$, where $N$ is the number of testing data. These pre-data processing will make the tests independent of signal amplitudes and easy to interpret.

If these correlation tests are satisfied, then the model residuals are random sequences and are not predictable from the model inputs. This provides an additional evidence of the validity of the identified model.

\section{AN ELNSS-BASED ONE-STEP-AHEAD PREDITIVE CONTROL}

\subsection{The one-step-ahead predictive control law}

Because the ELNSS model can approximate general nonlinear system and represent pseudo-linearity, many linear control design approaches, such as model reference adaptive control and predictive control, can be applied to the nonlinear system modeled by the ELNSS. In this section, a one-step ahead predictive control method is introduced. The configuration of ELNSS based closed loop control system is shown in Figure 2.

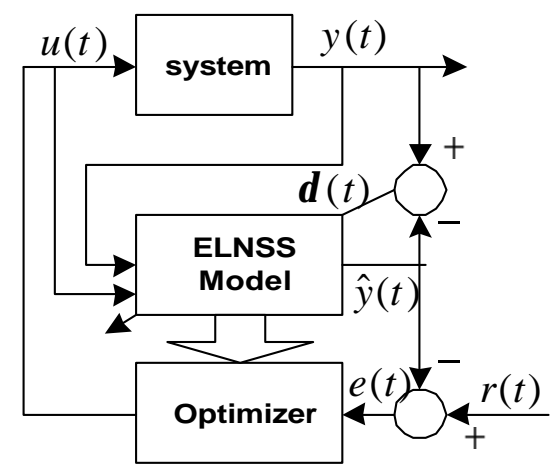

Fig.2 Configuration of ELNSS based closed loop control system

For a general nonlinear system (1), one can consider the following cost function

$$
J=\frac{1}{2}\left[y_{r}(t+1)-y_{p}(t+1)\right]^{2}+\lambda \Delta u^{2}(t)
$$

where $y_{r}(t+1)$ is the reference trajectory, $\lambda>0$ is a pre-specified control update weighting factor, $\Delta u(t)=u(t)-u(t-1)$, and $y_{p}(t+1)$ is the one-stepahead prediction produced from an ELNSS model. Using the gradient-search optimization approach, the output of the optimal controller that minimizes J can be obtained as

$$
u(t)=u(t-1)-\frac{\lambda}{1+\lambda^{2}} e(t+1) \frac{\partial e(t+1)}{\partial u(t)}
$$

where $e(t+1)=y_{r}(t+1)-y_{p}(t+1)$

The convergence of this control algorithm is summarized in the following theorem.

Theorem 2: If the parameter $\lambda>0$, the control algorithm given in (27) will be convergent.

Proof: Take the partial derivative of cost criteria (26) with respect to $t$, and it can be obtained that

$$
\frac{\partial J}{\partial t}=e(t+1) \frac{\partial e(t+1)}{\partial u(t)} \frac{\partial u(t)}{\partial t}+\lambda \Delta u(t) \frac{\partial u(t)}{\partial t}
$$

Approximately,

$$
\frac{\Delta J}{\Delta t}=e(t+1) \frac{\partial e(t+1)}{\partial u(t)} \frac{\Delta u(t)}{\Delta t}+\lambda \Delta u(t) \frac{\Delta u(t)}{\Delta t}
$$

Substituting (27) into (30), it can be further obtained that

$$
\Delta J=\frac{-\lambda}{\left(1+\lambda^{2}\right)^{2}} e^{2}(t+1)\left[\frac{\partial e(t+1)}{\partial u(t)}\right]^{2}
$$

It is obvious that $\Delta J$ is negative if $\lambda>0$, thus the control algorithm (27) is convergent.

Concerning the asymptotic stability of the ELNSS based closed-loop control system, if the one-step ahead predictive controller (27) is adopted, the following theorem should also hold. 
Theorem 3: Suppose the Lyapunov function is defined as follows,

$$
V=\frac{1}{2}\left[\left(1+\lambda^{2}\right) e^{2}(t+1)+\left(1+\lambda^{2}\right)^{2} \Delta e^{2}(t+1)\right]
$$

If one-step-ahead control (27) is used, the sufficient condition for the asymptotic stability of the closed loop system is

$$
0<\lambda<\frac{1}{f^{2}}
$$

where $\quad f=\frac{\partial e(t+1)}{\partial u(t)}$

Proof: Consider

$$
\begin{aligned}
& \frac{\partial V}{\partial t}=\left(1+\lambda^{2}\right) e(t+1) \frac{\partial e(t+1)}{\partial u(t)} \frac{\partial u(t)}{\partial t} \\
& +\left(1+\lambda^{2}\right)^{2} \Delta e(t+1) \frac{\partial e(t+1)}{\partial u(t)} \frac{\partial u(t)}{\partial t}
\end{aligned}
$$

Using the first order approximation, it can be obtained that,

$$
\begin{aligned}
& \Delta V=\left(1+\lambda^{2}\right) e(t+1) \frac{\partial e(t+1)}{\partial u(t)} \Delta u(t) \\
& +\left(1+\lambda^{2}\right)^{2} \Delta e(t+1) \frac{\partial e(t+1)}{\partial u(t)} \Delta u(t)
\end{aligned}
$$

from (27) and using the fact that

$$
\Delta e(t+1)=\frac{\partial e(t+1)}{\partial u(t)} \Delta u(t)=f \Delta u(t)
$$

it can be shown that

$$
\Delta V=\left(-\lambda+\lambda^{2} f^{2}\right) e^{2}(t+1) f^{2}
$$

If $0<\lambda<\frac{1}{f^{2}}$, then $\Delta V<0$, and the closed loop system will be asymptotically stable. Since approximation (27) is used, the closed loop stability is at least locally true.

\section{EXAMPLES}

Example 1: consider the affine nonlinear system:

$$
y(t)=\frac{u(t-1) y(t-1)}{1+y^{2}(t-1)}+u(t-1)
$$

The architecture of the ELNSS is 4-4-1, whose input is $I(t)=\left[x_{1}(t), x_{2}(t), x_{3}(t), u(t-1)\right]^{\mathrm{T}}$. Suppose that the initial EKF identification parameters are $P(0)=10^{5} \mathrm{I}$, $R(0)=2 * 10^{-5}$, and controller factor $\lambda=0.65$ with the reference trajectory $y_{d}(t+1)$ as

$y_{d}(t+1)=0.3 \sin (2 \pi t / 250)+0.2 \sin (2 \pi t / 100)$

then the closed loop response is shown in Figure3, where the mean squared of tracking error is $4.9 * 10^{4}$.

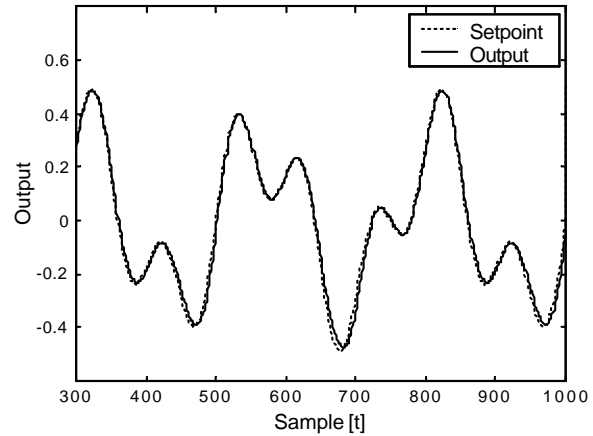

Fig 3. Closed loop system response of example 1

Example 2: Consider another nonlinear plant:

$$
y(t)=\frac{y(t-1) y(t-2)[y(t-1)+0.3]}{1+y^{2}(t-1)+y^{2}(t-2)}+u(t-1)
$$

The architecture of the ELNSS is again 44-1, and the initial EKF identification parameters are $P(0)=4 * 10^{5} * I, R(0)=2 * 10^{5}$ and the controller factor is selected as $\lambda=0.4$. The whole training process has used 1000 iterations. In order to overcome the inaccuracy of the ELNSS model, the on-line feedback correction method is adopted to give

$$
y_{p}(t+1)=y_{m}(t+1)+h\left(y_{p}(t)-y_{m}(t)\right)
$$

where $h$ is an error correction factor. In this simulation $h=1$. The reference trajectory $y_{d}(t+1)$ is selected as

$$
\begin{aligned}
& y_{d}(t+1)= \\
& \left\{\begin{array}{lc}
0.2 \sin (2 \pi t / 250)+0.3 \sin (2 \pi t / 100) & 0<t<500 \\
0.04 \sin (2 \pi t / 25)+0.2 \sin (2 \pi t / 250) & 500<t<1000
\end{array}\right.
\end{aligned}
$$

The closed loop response is shown in Figure 4, and the mean squared of tracking error is $5.72 * 10^{-4}$.

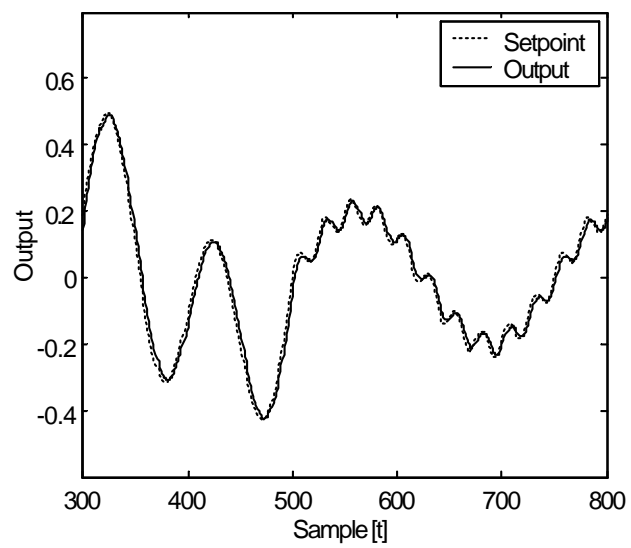

Fig 4. Closed loop system response of example 2

\section{CONCLUSION}

In this paper, an extended linearized neural state space model is developed, which can model a class of nonlinear dynamic systems. Since the ELNSS exhibits pseudo-linearity of the plant, most linear 
system controller design approaches can be extended to apply to the ELNNS models. In particular, the ELNSS is an approximation of Volterra basis neural networks in some cases. Therefore, the ELNSS is suitable to model the system with polynomial characteristics. The extended Kalman filtering based learning algorithm is adopted to estimate the parameters of the ELNSS. The existence of ELNSS as a universal model of the nonlinear dynamic discrete system and the convergence of learning process were established. Indeed, the convergence is guaranteed when the learning rate is correctly chosen according to the rule in this paper.

The one-step-ahead predictive control approach based on the ELNSS was proposed. The stability issues based on well-known Lyapunov theory were investigated, and the corresponding sufficient conditions for the local asymptotic stability of the ELNSS based control closed-loop system were derived. In fact the approach used in the stability analysis is also effective for that of the MLP based control system. However, difficulty remains in the stability analysis for general nonlinear control systems. Further investigation is therefore needed in this respect.

The satisfactory control performances of simulation examples showed that the presented ELNSS based control scheme could handle some nonlinear process control problems.

\section{REFERENCES}

Billings S. A. and Voon W. S. F. (1986), Correlation-based model validity tests for nonlinear models, Int. J. Contr., vol. 44, pp. 235-244.

Friendland, B. (1996) Advanced Control system design, Prentice-hall, Englewood Cliffs, NJ Chap. 4

Gintaras V. Puskorius and Lee Feldkamp, (1994), Neurocontrol of Nonlinear Dynamical Systems with Kalman Filter Trained Recurrent Networks, IEEE Trans. On Neural Networks, Vol. 5, No. 2, pp. 279-297.

Liang Jin, Madan M. Gupta, Peter N. Nikiforuk (1999), Dynamic Recurrent Neural Networks for Approximation Of Nonlinear Systems, Proc. $14^{\text {th }}$ IFAC, Vol. K, pp. 45-50.

Liu Y. N and Feng C. B. (1995), The Possibility using neural network to model a nonlinear discrete dynamic system, Control Theory and Application, (in Chinese), 12(4), pp. 100-106.

Ljung, L. (1987). System Identification. Theory for the User. Prentice Hall, New Jersey.

Pavel Zite et al (1999), Neural Network Processing of Observer Generated Residuals in Fault Diagnosis of Time Delay Systems, Proc. $14^{\text {th }}$ IFAC, Vol. P, pp. 79-84.
Suykens, J. A. K., B. DeMoor, and J. Vandewalle (1995), Nonlinear System Identification Using Neural State Space Models, Applicable to Robust Control Design, Int. J. Control, Vol. 62, No. 1, pp. 129-152.

Tan Yonghong and Cauwenberghe A. V. (1996), A. Nonlinear One-step-ahead control Using neural Networks: control Strategy and Stability Design, Automatica, 32(12), pp. 1701-1706.

Wang, Y.J., G. Fernholz and S. Engell, (1998a), A Second Order Recursive Prediction Error Algorithm for Diagonal Recurrent Neural Networks, Proc. of IEEE Conf. On Control Application 98, pp. 172-176, Trieste, Italy, Sept.1-4, 1998.

Wang, Y.J., G. Fernholz and S. Engell, (1998b), A New Pseudolinear Neural Network for a King of Nonlinear Dynamic Systems Modeling. Proc. International workshop on Advanced Black-Box techniques for Nonlinear Modeling, pp. 184-189, Leuven, Belgium, July, 8-10,1998.

Wang, Y. J., G. Fernholz and S. Engell (1999a), Pseudolinear Neural Networks Based Nonlinear System Modeling and Control. Proc. 14 $4^{\text {th }}$ IFAC, Vol. K, pp. 355-360.

Wang Yongji, Wang H (1999b), A Nonlinear Model Predictive Control based on Pseudolinear Neural Networks, Proc. of ECC 99, pp. 1057-1062

Zamarreno J. M., P. Vega (1998), State space neural network. Properties and application, Neural Networks, 11, pp. 1099-1112.

Zamarren J. M., P. Vega, L. D. GarcmHa et al (2000), State-space neural network for modelling, prediction and control, Control Engineering Practice, 8, pp. 1061-1075.

Zhao Hong, John Guiver, Guillermo Sentoni (1998), An Identification Approach to Nonlinear State Space Model for Industrial Multivariable Model Predictive Control. Proc. of ACC, pp. 796-800.

Zhao Hong, J. Guiver, R. Neelakantan et al (1999a), A Nonlinear Industrial Model Predictive Controller Using Integrated PLS and Neural Net State Space Model, Proc. $14^{\text {th }}$ IFAC, Vol. N, pp. 13-18.

Zhao Hong, John Guiver, Guillermo Sentoni (1999b), An Identification Approach for Industrial Nonlinear Model Predictive Control, Proc. $14^{\text {th }}$ IFAC, Vol. N, pp. 205-210. 